$\triangle C T A$ NEOPHILOLOGICA

UDK: 821.112.2.09-2Tabori G.:341.485(=411.16)

DOI: 10.4312/an.50.1-2.173-187

\title{
Die Darstellbarkeit des Bösen: Zu George Taboris Holocaust-Literatur
}

\author{
Špela Virant
}

\begin{abstract}
Das künstlerische Schaffen George Taboris lässt sich nicht nur einer Kunstgattung oder einer Nationalliteratur zuordnen, doch gerade seine Interdisziplinarität und sein Kosmopolitismus leisteten im ausgehenden 20. Jahrhundert einen wichtigen Beitrag zur europäischen Kultur. Im Beitrag wird sein Stück Mein Kampf aus einer interkulturellen Perspektive im Kontext der jüdisch-deutschen Beziehungen in Österreich der Nachkriegszeit betrachtet. Die Uraufführung des Stücks, das gegen die Mythisierung Hitlers und die Viktimisierung der Juden anschreibt, fand in einer Zeit statt, in der der Opfer-Status des österreichischen Staates ins Wanken geriet und das Judentum in Wien neues Selbstbewusstsein erlangte.
\end{abstract}

Schlüsselwörter: George Tabori, Dramatik, Holocaust-Literatur, Juden in Wien, Ironie 
Als sich im Jahr 2014 George Taboris Geburtstag zum hundertsten Mal jährte, gab es keine große Feier anlässlich des Jubiläums. Es mag sein, dass der 2007 verstorbene Künstler noch nicht lange genug tot war, um sich an ihn zu erinnern. Der Grund für das Schweigen könnte jedoch auch an der Unklarheit bezüglich der Zuständigkeiten liegen, denn Tabori, eigentlich György Tábori, lässt sich schwer einordnen. Als Theaterregisseur berühmt geworden, wurde seine Arbeit zunächst von der Theaterwissenschaft aufgearbeitet, erst spät fand sein schriftstellerisches Werk Beachtung in der Literaturwissenschaft. Noch schwieriger als die Zuständigkeit der Disziplinen zu bestimmen, ist seine Zuordnung zu einer nationalen Literatur. Tabori, 1914 in Budapest in einer jüdischen Familie geboren, machte eine Ausbildung im Hotelgewerbe in Berlin, emigrierte nach London, arbeitete als Auslandskorrespondent in Bulgarien und dem Nahen Osten, erhielt die britische Staatsbürgerschaft und arbeitete während des Zweiten Weltkriegs für den britischen Nachrichtendienst, zog nach dem Krieg in die USA, zunächst nach Hollywood, dann nach New York, kehrte 1971 zurück nach Europa, arbeitete in Deutschland und Österreich, schließlich starb er 2007 in Berlin. Seine ersten literarischen Texte verfasste und veröffentlichte er in englischer Sprache. Die in Europa entstandenen Stücke sind jedoch nur in deutscher Übersetzung erschienen und in deutscher Sprache uraufgeführt worden, so dass sie praktisch als Originale gelten können. Die meisten davon übersetzte Ursula Grützmacher-Tabori, die dritte seiner vier Ehefrauen. ${ }^{1}$ Taboris literarisches Werk lässt sich deshalb nur schwer einer Nationalliteratur oder einem Sprachraum zuordnen.

Obwohl im 21. Jahrhundert multikulturelle Ansätze, postkoloniale Studien und Migrationsliteraturforschung in die Literaturwissenschaft Einzug fanden, orientiert sich die Literaturgeschichtsschreibung immer noch an Nationalliteraturen. Die Migrationsliteratur ist zum Gegenstand germanistischer Literaturwissenschaft geworden, insoweit Migranten in deutscher Sprache schreiben und sich ihre Texte der deutschsprachigen Literatur zuordnen lassen. Zwischen den Konzepten von Nationalliteratur und Weltliteratur hat sich in der Literaturgeschichtsschreibung nie ein multikultureller und multilingualer Raum aufgetan. Taboris Werk lässt sich aus zwei Gründen nicht der sogenannten Migrationsliteratur zuordnen: Erstens weil diese Bezeichnung die Thematisierung der Migration vorausgesetzt, die nicht sein Thema war, zweitens wegen der Sprache, in der seine Texte verfasst worden sind. Auch seine Biographie suggeriert für ihn eher den altmodischen Begriff des Kosmopoliten. Doch gerade weil sich seine Arbeit einer Einordnung entzieht, leistete sie einen wichtigen Beitrag zur Kulturgeschichte im mitteleuropäischen Raum. Im Folgenden soll dieser Beitrag am Beispiel seines 1987 im Akademietheater in Wien uraufgeführten Stücks Mein

1 Zu den biographischen Angaben über George Tabori vgl. Feinberg 2003. 
Kampf dargestellt werden. Das Stück wird im Rahmen seines Gesamtwerks und der sozial-politischen Umstände in Österreich zur Zeit seiner Uraufführung verortet, wobei besondere Aufmerksamkeit nicht einer klar definierbaren nationalen Literatur und Kultur, sondern den interkulturellen jüdisch-österreichischen Beziehungen gewidmet wird.

Taboris erste, in englischer Sprache geschriebene Stücke Flight into Egypt (1953) und The Emperor's Clothes (1953) waren nicht erfolgreich, doch er widmete sich danach trotz des Misserfolgs intensiv dem Theater und wurde Mitglied des von Lee Strasberg geleiteten Actor's Studio, was auch später seine Arbeit prägte. Den ersten großen Erfolg brachte die deutsche Erstaufführung des Stücks Die Kannibalen in Berlin 1969, während die englischsprachige Uraufführung ein Jahr zuvor in New York relativ unbeachtet blieb. Das Stück, in dem er die Erinnerung an seinen in Auschwitz getöteten Vater Cornelius verarbeitet, ist der Beginn seiner Auseinandersetzung mit dem Holocaust, der in seinen späteren Prosatexten und Theaterstücken immer wieder thematisiert wird, so auch im Stück Mein Kampf (1987). Seine Werke können durchaus zur Holocaust-Literatur gezählt werden, doch gehört er nicht zu den Zeitzeugen im engeren Sinne, denn er berichtet nicht über eigene Erfahrungen aus den Konzentrationslagern, er selbst war nie interniert. Er berichtet über seine Erfahrungen als Familienangehöriger der Opfer, über die Schwierigkeit, mit dem Leid geliebter Menschen und der Trauer umzugehen, ohne dieses Leid selbst erfahren zu haben. „Tabori, dessen Vater in Auschwitz starb, übernimmt weder in Schriften noch in (Selbst)Inszenierungen die Perspektive der Opfer, aber er vergegenwärtigt ihr Leiden." (Huth 2008: 120) In seinen Werken spricht er über das Sprechen über den Holocaust, um so auch über den Holocaust sprechen zu können. Dies ist ein Weg, Tabus zu brechen:

Bis zur Aufführung von Taboris Kannibalen und wieder lange Zeit danach herrschte auf Deutschlands Bühnen und in den Bühnenmanuskripten entweder eine Fixierung auf die Täterfiguren oder aber ein Darstellungs-Tabu, das es verbot, differenziert gestaltete jüdische Figuren an den Schauplätzen ihres Leidens agieren zu sehen. (Stümpel 2000: 49-50)

Das Stück Die Kannibalen zeichnet sich durch eine komplexe dramaturgische Struktur aus, die für die Schauspieler eine Herausforderung darstellt. Sie übernehmen die Rollen von Söhnen, die die Figuren ihrer im Konzentrationslager Auschwitz getöteten Väter spielen, um sie so besser zu verstehen und das Trauma der Überlebenden zu überwinden. Die Geschichte der Väter, die im Lager spielt, ist makaber. Der Lagerinsasse Puffi isst heimlich ein Stück Brot, die Mitgefangenen ertappen ihn dabei und töten ihn unabsichtlich. Da sie sehr unter dem Hunger leiden, wollen sie ihn kochen und aufessen, aber einer der Gefangenen, der als 
Onkel bezeichnet wird, lehnt es ab. Er plädiert dafür, trotz der Not die Würde zu bewahren und er zeigt ihnen den Weg, durch das Erzählen und das Rollenspiel ihre Menschlichkeit aufrecht zu erhalten und sich nicht zu Menschenfressern machen zu lassen. Doch auch er stirbt bei einem Tumult. Ein Aufseher zwingt die Lagerinsassen das von ihnen gekochte Menschenfleisch zu essen. Einige, die vom Onkel umgestimmt wurden, weigern sich nun und werden in der Gaskammer getötet. Nur zwei gehorchen und überleben.

Tabori stellt dadurch die Zuschreibung und Verfestigung der Rollen von Opfer und Täter in Frage. Er zeigt, dass Opfer auch Täter sein können und dass sich Menschen nicht auf eine Rolle reduzieren lassen. „Taboris Juden sind brutal, grotesk und bisweilen auch geschmacklos. Dadurch gibt er ihnen ihre Menschlichkeit wieder, die ihnen durch Sentimentalisierung und Sakralisierung ihrer Leiden entzogen wurde." (Roth 2003: 141) Mit der Opfer-Problematik beschäftigte sich Tabori auch in anderen Stücken, die vor dem Hintergrund des Rassismus in den USA spielen oder die Problematik des Vietnam-Krieg thematisieren. Am deutlichsten wird dieses Thema jedoch in dem Stück Weisman und Rotgesicht (1991) ausgearbeitet, in dem es sich herausstellt, dass der Indianer Rotgesicht eigentlich der Halbjude Goldberg ist, der schließlich die Rolle eines Cowboys übernimmt. Tabori relativiert dadurch nicht das Leid der Opfer, sondern versucht eine erneute Viktimisierung durch Rollenzuschreibung zu verhindern. Dabei wird jedoch nicht davon ausgegangen, dass sich die Rollen abschütteln lassen, um den 'wahren' Menschen zum Vorschein zu bringen, sondern vielmehr von der Wandelbarkeit und Widersprüchlichkeit des Menschen, der nacheinander oder gleichzeitig mehrere Rollen spielen kann. Eben das müssen auch die Schauspieler in dem Stück Die Kannibalen tun. Der Wechsel zwischen verschiedenen Spielebenen, der durch Kommentare und Autoreflexionen begleitet wird, verweist auf die seit dem Holocaust ausgiebig diskutierte Frage nach der Darstellbarkeit der Grausamkeiten, die sich im 20. Jahrhundert in Lagern, Kriegen und Genoziden ereigneten. Auf der autoreflexiven Ebene wird in Taboris Texten dem Erzählen und dem schauspielerischen Nacherleben eine heilende Wirkung zugesprochen, die, auch wenn das Dargestellte nie authentisch vermittelt werden kann, sowohl dem Einzelnen wie auch der Gesellschaft ein Weiterleben ermöglicht.

Das Gegenstück zu Den Kannibalen stellt das Stück Mutters Courage (1981, Uraufführung 1979) dar, und zwar als Versuch, die Geschichte von Taboris Mutter nachzuerzählen, die sich der Internierung in ein Lager entziehen konnte und den Krieg überlebte. In dem Stück, dem ein Prosatext vorausging, erzählt der Sohn die Geschichte der Mutter, die es wagte, einem deutschen Offizier in die Augen $\mathrm{zu}$ sehen und zu behaupten, dass sie eigentlich einen Schutzpass habe und nicht ins Lager gehöre, wodurch sie gerettet wird. Die Mutter hört der Erzählung zu, ergänzt sie und korrigiert nach Bedarf ihren Sohn. Diese Einwürfe führen auch 
hier eine autoreflexive Ebene in die Erzählung ein, die die Möglichkeiten des Erinnerns und die Funktionen des Erzählens auslotet. Die erzählte Geschichte selbst zeigt, wie sich ein System, das auf Entmenschlichung basiert, durch Mut zur Menschlichkeit unterlaufen lässt.

Tabori beschäftigte sich immer wieder einerseits mit dem Holocaust und der jüdischen Identität, andererseits mit deren Darstellbarkeit in der Kunst wie auch mit den Funktionen der Erzählkunst und des Theaters im Allgemeinen. Beide Themenschwerpunkte werden im Stück Goldberg-Variationen (1994, Uraufführung 1991) meisterhaft verflochten. Das Stück spielt im Theater, es zeigt die Entstehung einer Inszenierung und endet, als sich der Vorhang für die Premiere dieser fiktiven Inszenierung hebt. Mr. Jay, der fiktive Regisseur, der mit Jahwe assoziiert wird, will mit Hilfe seines Regieassistenten Goldberg, der den Holocaust überlebte, die Bibel auf der Bühne dramatisch darstellen. Diese Konstellation ermöglicht es Tabori all seine Kunstfertigkeit bei der Dialoggestaltung auszuspielen und mit leichter Hand, die vor Witz, Ironie, Zotte und Kalauer nicht zurückschreckt, komplexe Themen zu vermitteln.

In Taboris Gesamtwerk, in dem, wie aus der kurzen Darstellung ersichtlich, die Auseinandersetzungen mit dem Holocaust und der jüdischen Identität immer wieder einen Schwerpunkt bilden, nimmt sein Stück Mein Kampf(1987), das zu seinem meistgespielten Text wurde, eine besondere Stellung ein, und zwar nicht nur wegen der Thematik und der Ausgestaltung des Textes, sondern auch wegen des zeitgeschichtlichen Kontexts, in dem die Premiere stattfand. Im Untertitel wird Mein Kampf als Farce bezeichnet. ${ }^{2}$ Der Aufbau ist, verglichen mit anderen Texten Taboris, relativ konventionell. Die Gliederung in fünf Akte erinnert an die traditionelle Dramenstruktur. Diese formelle Anlehnung an die Dramentradition hebt das Provokative des Stücks noch stärker hervor. Provokativ ist schon der Titel, der auf Adolf Hitlers Buch verweist, das, mit Ausnahme der 2016 erschienenen kritischen Ausgabe, in Deutschland und Österreich nicht nachgedruckt und verkauft werden darf. Provokativ ist auch der humorvolle und ironische Ton, in dem das Stück über die traumatische Vergangenheit spricht. ${ }^{3}$ Zwar wurde Hitler bereits 1940 von Charlie Chaplin in dem Film Der große Diktator parodiert, doch im deutschsprachigen Raum war im Kontext der Vergangenheitsbewältigung die Verbindung von Holocaust mit Humor bis zur Premiere von Taboris Stück praktisch unmöglich, da sie als despektierlich gegenüber den Opfern galt und den Verdacht einer Nähe zu Holocaustleugnern und Neonazisten weckte. Dieser

2 In der Forschungsliteratur werden auch Begriffe wie theologischer Schwank (Pott 1997: 248269), Volksstück (Thunecke 1993: 246-272) und Tragödie (Liessmann 1997: 81) als mögliche Gattungsbezeichnungen in Erwägung gezogen.

3 Zur Komplexität des Humors in diesem Stück vgl. Höyng 1998. 
Verdacht kam hier wegen seiner Biographie und seiner vorherigen literarischen Texte nicht auf. Die Gräuel werden in seinem Stück auch nicht verharmlost, sondern durch die Kontraste und Wendungen noch suggestiver vermittelt.

Das Stück zeigt die Genese des großen Diktators. Doch der fiktive Hitler wird in der Personenkonstellation zu einer Nebenfigur degradiert. Die Hauptfigur ist Schlomo Herzl, ein jüdischer Bibelverkäufer, dem zur Seite steht der jüdische Koch Lobkowitz, der an einem Gott-Komplex leidet und vielleicht auch tatsächlich ein Gott ist. ${ }^{4}$ Die Geschichte spielt am Beginn des 20. Jahrhunderts in Wien, im Männerasyl in der Blutgasse, wo alle drei, von Misserfolgen geplagt, Zuflucht finden. ${ }^{5}$ Der dramatische Konflikt erwächst aus den Verwicklungen um Schlomos vermeintliche Autobiographie, die er als „Mein Kampf" betiteln will, aber nie wirklich schreibt. Am Beginn des Stücks, das auch als Inversion des Passah-Festes interpretiert werden kann, indem statt der symbolischen Neugeburt des jüdischen Volkes der Holocaust ankündigt wird (vgl. Haas 2000: 161-198), kümmert sich Schlomo um den jungen Hitler, der nach seinem Scheitern an der Kunstakademie sehr unbeholfen ist. Der Jude Schlomo macht Hitler zu dem, den die Zuschauer aus der Geschichte kennen: Er gestaltet seine Frisur und seinen Bart und er zeigt ihm den Weg in die Politik. Er beschützt ihn vor Frau Tod, die nach ihm sucht, doch es stellt sich heraus, dass seine Sorge unbegründet ist, da sie ihn nur als Zuarbeiter braucht. Im letzten Akt wird die Stimmung düster. Hitler, der inzwischen bereits Erfolge feiert, sendet seine Gehilfen aus, um nach Schlomos Buch zu suchen, da er fürchtet, es könnte zu viel über ihn verraten. Die Suche bleibt erfolglos, aber die Gehilfen streichen die Wände braun und hinterlassen eine Vorahnung kommender Ereignisse, über die Schlomo zwar nicht Bescheid weiß, wohl aber Lobkowitz und die Zuschauer. Auf Lobkowitzs Anraten hin, der den Hunger der Konzentrationslager vorausahnt, verspeist Schlomo das geliebte Huhn, das von Hitlers Gehilfen geschlachtet wurde.

Neben der Literarisierung von Hitler verwendet Tabori in diesem Stück auch Anspielungen an historische Persönlichkeiten - Schlomo Herzel erinnert an Theodor Herzel, Hitlers Gehilfe Himmlischst an Heinrich Himmler. Die Personifizierung des Todes wird durch die Differenz zwischen dem grammatikalischen

4 „Lobkowitz ist nicht Gott, aber das Spiel mit Lobkowitz ermöglicht es Herzl, mit Gott, dem echten, zu sprechen." (Scholz 2002: 112). Zur ausführlichen Diskussion des Verhältnisses zwischen Lobkowitz und Gott vgl. Scholz (2002: 111-116).

5 Ivar Oxaal kommentiert Hitlers Aufzeichnungen, in denen er erzählt, er habe die Juden in Wien anfangs gar nicht bemerkt: "This was a tricky way of attempting to make amends to the reader for his earlier lack of racial awareness, because he knew, having himself lived in a men's hostel north of the canal on Meldemannstrasse from December 1909, that the Brigittenau district contained the major concentration of the highly visible, impoverished, Jews from Galicia." (Oxaal 1987: 28) Die Naivität von Taboris junger Hitlerfigur steht nicht völlig im Widerspruch mit disem historischen Bild. 
Geschlecht und dem Geschlecht der Figur verfremdet. Schlomos junge Geliebte Gretchen erinnert an die literarische Figur aus Goethes Faust, aber auch an Margarete aus Paul Celans Todesfuge. ${ }^{6}$ Dadurch werden auf der Ebene der Namensgebung historische Fakten mit Fiktion vermischt, was auch auf der Ebene der dargestellten Geschichte passiert, in der hinlänglich bekannte Daten aus Hitlers Biographie mit Fiktion verwoben werden. Dadurch entstehen zahlreiche komische Effekte, vor allem aber eine durchaus ernsthafte Hinterfragung der Geschichtsschreibung, die zwar objektive historische Wahrheiten vermitteln will, durch die Herstellung von Zusammenhängen und ihre Versprachlichung jedoch auch Interpretationsprozesse verwendet, wie sie in der Literaturproduktion und -rezeption zur Herstellung von Fiktion gebräuchlich sind. Taboris autoreflexive Texte, besonders beide Stücke, in denen er sich den Lebensgeschichten seiner Eltern anzunähern versucht - also Die Kannibalen und Mutters Courage -, vermitteln das Ausloten der Möglichkeit, wie Fiktion sich der historischen Wahrheit nähern kann, und zwar nicht durch verlässliche Vermittlung von Daten, die nur rational verarbeitet werden, sondern durch das Angebot, empathisch die Geschichte nachzuerleben. Der Holocaust ist ein Teil der Geschichte des 20. Jahrhunderts, der nur durch Zahlen und Daten in seiner Bedeutung und seinen Folgen nicht erfassbar ist. Erst ein empathischer, narrativer Vorgang macht die traumatischen, der Heilung bedürfenden Nachwirkungen sowie die Warnungen vor möglichen Wiederholungen nachvollziehbar. Tabori vermittelt in seinen Texten nicht nur negative Gefühle, sondern stellt ihnen immer auch Liebe und Sympathie gegenüber, sowie den Mut, sie im Alltag zu leben, auch auf die Gefahr hin, dass dadurch Figuren wie Schlomo naiv oder beschränkt wirken. Schlomos freundschaftliche Zuneigung zu Hitler wird missbraucht und er sitzt am Ende des Stücks eindeutig als Verlierer vor dem geschlachteten Huhn, dem Symbol seiner liebenden Hingabe. Das Stück endet trotzdem versöhnlich kokett, wenn auch nicht ohne Ironie: Gretchen küsst Schlomos Nase, den Körperteil, der von den Nationalsozialisten als besonderes Rassenmerkmal der Juden oft karikiert wurde (vgl. Tabori 1994: 203).

Taboris Theater und Literatur wurden im Kontext der Diskussion über die Darstellbarkeit des Holocaust bereits eingehend untersucht. Diese Diskussion wurde 1951 von Theodor W. Adornos Aufsatz Kulturkritik und Gesellschaft mit dem Diktum „nach Auschwitz ein Gedicht zu schreiben, ist barbarisch“ angestoßen (Adorno 1977: 30). Als Reaktion darauf haben sich drei Grundpositionen gebildet: Die erste vertritt eine Art Darstellungsverbot; die zweite, in Anschluss

6 Eine ausführliche Studie zur Intertextualität bei Tabori bietet Alice Huth, die sich besonders auf Bezüge zu Shakespeare, Lessing und Kafka auf der Basis von Renate Lachmanns Theorie der Intertextualität konzentriert, und zwar ,als Formen ästhetischer Auseinandersetzung mit Auschwitz"(Huth 2008: 120). 
an Adornos spätere Antwort auf Enzensbergers Widerspruch, vertritt die Position, dass ein literarisches Engagement trotz der grundsätzlichen Undarstellbarkeit notwendig sei, um nicht in Zynismus zu verfallen; die dritte hingegen verweist auf eine Literatur, die keine Repräsentation des Leids ist, sondern sich hermetisch gegen eine realistische Widergabe von Wirklichkeit sperrt und nicht als Abbild, sondern als Ausdruck des Leids wahrnehmbar ist (vgl. Adorno 1973: 355). Dies kann nur durch eine Desautomatisierung der Wahrnehmung erreicht werden: „Künstlerisch zu erreichen sind die Menschen überhaupt nur noch durch den Schock“ (Adorno 1970: 476). Auf eine vierte Position verwies Ruth Klüger, die nicht von dem Kunstrezipienten ausgeht, sondern von dem Autor, der die Literatur braucht, „um sich seelisch über Wasser zu halten“ (Klüger 1992: 126). Tabori integrierte all diese Bedenken in seine Texte, ging nicht auf die bereits etablierte Hermetisierung ein, noch griff er zurück auf pure Abbildung, sondern fand neue Möglichkeiten, das Theaterpublikum zu schockieren. ${ }^{7}$ Durch Humor und Ironie, durchflochten mit Autoreflexion, schafft er eine lockere Atmosphäre, die plötzlich in Düsternis umkippen kann. Schon dieses Umkippen schockiert. Der zweite Schock kommt, wenn sich das Publikum seiner eigenen Rezeptionsvorgänge bewusst wird. Was von der Tabori-Forschung bisher jedoch weniger beachtet wurde, ist die Interpretationsmöglichkeit, dass Taboris Kombination von Humor und Autoreflexion die Debatte über die Darstellbarkeit des Holocaust selbst ironisiert und sie in die Tradition dessen einreiht, was Adorno in dem bereits erwähnten Aufsatz Kulturkritik und Gesellschaft als die absolute „Verdinglichung, die den Fortschritt des Geistes als eines ihrer Elemente voraussetzte“ (Adorno 1977: 30), bezeichnete. Tabori gelang es, die Problematik des Holocaust und seiner Darstellbarkeit aus den in sich verschlossenen akademischen Debatten wieder in die Öffentlichkeit zu bringen. „In Taboris Theaterarbeit können die Witze zwar die Katastrophe überrumpeln, aber sie bringen das Trauma der Erinnerung nicht zum Schweigen." (Dahlke 1997: 154) Taboris literarische Aufarbeitung der deutsch-jüdischen Geschichte, die die Traumata nicht leugnet oder verdrängt, aber auch nicht auf Schuldzuweisungen und unversöhnlichen Vorwürfen insistiert, stieß in Wien in den späten 1980er Jahren auf offene Ohren, was in Bezug auf die Geschichte keineswegs selbstverständlich war. Die Inszenierung des Stücks im Akademietheater des Burgtheaters, also in der zentralen kulturellen Institution der spätbürgerlichen Selbstinszenierung, markiert einen Wendepunkt in der Nachkriegsgeschichte der deutsch-jüdischen Beziehungen, die in Österreich noch problematischer war als in Deutschland.

7 Berghahn sieht in Taboris „Heiterkeit der Verzweiflung“ eine Nähe zu Adornos ästhetischer Theorie, zweifelt jedoch, ob „Adorno der polemischen Komik Taboris in allen Stücken zugestimmt hätte“ (12). 
Schon ein flüchtiger Blick auf die Statistik des Wiener Judentums zeigt die wichtigste Ursache für diese problematische Beziehung. Im Jahr 1848 lebten in Wien 4.000 Juden, im Jahr 1860 waren es 6.200, was etwa 2,2 \% der Gesamtbevölkerung betrug, im Jahr 1923 schon 201.000, d. h. 10,8 \% der Gesamtbevölkerung (vgl. Schubert 2008: 79). Das Bildungsniveau war recht hoch: „Im Studienjahr 1889/90 waren an der Universität Wien $22 \%$ der Studierenden an der juridischen Fakultät Juden, an der medizinischen Fakultät $48 \%$ und an der philosophischen Fakultät 15 \%.“ (Ebd.: 80) Doch „Ende 1945 zählte die Israelitische Kultusgemeinde keine 5.000 Mitglieder“ (Embacher 1995: 21).

Am Beginn des 20. Jahrhunderts stammten etwa 40 Prozent der Wiener Bevölkerung aus nicht-deutschen Gebieten der Habsburger Monarchie (vgl. Rozenblit 1992: 13). Etwa 25 Prozent der jüdischen Bevölkerung stammte aus Galizien, die meisten jedoch waren assimiliert, gebildet und sozial und finanziell besser gestellt (vgl. ebd.: 12). Marsha L. Rozenblit sieht darin den Grund, dass in Wien radikaler deutscher Nationalismus nie populär wurde (vgl. ebd.: 13).In ihrer umfangreichen Studie zu den Juden Wiens in der Zeit zwischen 1867 bis 1914 stellt sie fest, dass sie in dieser Zeit bestrebt waren, „sowohl eine jüdische als auch eine europäische Identität herauszubilden, und sie waren ehrlich davon überzeugt, daß ihnen dies schließlich auch gelingen würde“ (Rozenblit 1989: 195). Steven Beller, der die Epoche bis 1938 untersuchte, vertritt die These, dass sich nicht nur die Juden der österreichischen Kultur anpassten, sondern auch die österreichische Kultur stark von ihnen geprägt wurde (vgl. Beller 1989: 70). Er macht jedoch darauf aufmerksam, dass man nicht vereinfachend von einem einheitlichen Einfluss ausgehen kann, sondern von einer Vielfalt von unterschiedlichen Arten, wie die jüdische Herkunft Einzelne in ihrer Ideenwelt beeinflusst hat (vgl. ebd.: 82-83). Er stellt fest, dass um 1900 die jüdische intellektuelle Elite die Modernität der Wiener Kultur entscheidend bestimmte: "it was indeed its Jews which made Vienna what it was in the realm of modern culture" (ebd.: 244). Hervorzuheben ist auch die Bedeutung der Juden für das österreichische Theater der Zwischenkriegszeit, vor allem die Bedeutung Max Reinhardts und des Yiddischen Theaters in Wien (vgl. Silverman 2012: 172, 142).

Nach dem Zweiten Weltkrieg fehlte nicht nur im Theater, sondern in ganz Österreich jene Schicht, die am Beginn des Jahrhunderts Wien zu einer modernen, weltoffenen Stadt machte, zum europäischer Zentrum von Kunst und Kultur. Der Germanist Harry Zohn besuchte 1956 Wien nach langer Zeit und notierte:

Heute befinden sich die Wiener Juden nicht mehr im Hauptstrom des Stadtlebens, sie können nicht länger den so wichtigen 'Sauerteig' beistellen; im praktischen Sinn also ist das Nachkriegs-Wien eine 'Stadt ohne Juden'. Ich meinerseits hege keinen Zweifel, daß der Provinzialismus, welcher im täglichen 
Leben wuchert, die Verrohung des Geschmackes, die Verminderung des Kosmopolitismus der Stadt, in gewissem Maße der Dezimierung der jüdischen Bevölkerung zuzuschreiben ist. (Zohn 1956: 14).

Dieses von Zohn beobachtete Absinken in die Provinzialität war für die Stadt sicherlich eine Katastrophe, doch die wenigen in Wien verbliebenen oder zurückgekehrten Juden hatten größere Probleme als die Verrohung des Geschmacks, ${ }^{8}$ dazu gehörten: der „ ab 1948 immer offener auftretende Antisemitismus“ (Embacher 1995: 87); die Spannungen zwischen den alt-österreichischen assimilierten Juden und den neu zugereisten Ost-Juden sowie zwischen Anhängern unterschiedlicher politischer Parteien und Ideologien; die Auswirkungen des problematischen Status Österreichs als erstes Opfer von Nazi-Deutschland, der es dem Staat ermöglichte, keine Wiedergutmachung zu zahlen. ${ }^{9}$ Erst Anfang der 1990er Jahre begann sich die Situation in dem Land, in dem nie eine gründliche Entnazifizierung stattgefunden hatte (vgl. Wistrich 1992: 241), zu ändern: „Im Juli 1991 gab Bundeskanzler Franz Vranitzky im Rahmen einer Erklärung zu Jugoslawien eine vor allem im Ausland vielbeachtete Stellungnahme über Österreichs Mitverantwortung im 'Dritten Reich' ab." (Embacher 1995: 260)

Die Demütigungen und Beleidigungen, die die Juden in Österreich durch den Antisemitismus (vgl. ebd.: 95) und die Freisprüche für Nazi-Verbrecher erfahren mussten (vgl. ebd.: 252), schwächten ihr Selbstbewusstsein. Erst in den 1980er Jahren kommt es zu einem Generationswechsel: „Die überalterten und in die Minderheit geratenen 'Wiener Juden' sahen sich allmählich von einer kritischen und selbstsicheren, jüngeren Generation abgelöst." (Ebd.: 256) Eine Reihe von Veranstaltungen der Israelitischen Kultusgemeinde in der ersten Hälfte der 1980er Jahre stärkte das neue jüdische Selbstbewusstsein, ${ }^{10}$ das sich von der Waldheim Affäre 1986, die als Katalysator für eine in breiteren Gesellschafsschichten stattfindende Vergangenheitsbewältigung gesehen werden

8 Vgl. auch die literarischen Darstellungen dazu: „Diese Kombination von jüdischer Vergangenheit und judenloser Gegenwart - und dazwischen die Geschichte des Holocaust - verursacht ein Spannungsverhältnis, das zugleich eines der Hauptthemen in den Romanen jüngerer jüdischer Autoren ist: der Dualismus zwischen Juden und Nichtjuden, der zum übergroßen Teil in Kommunikationsunfähigkeit gipfelt." (Kunne 2000: 274)

9 Vgl. dazu auch Robert Knight, der auf das Spannungsverhältnis zwischen der Selbstdarstellung des Staats als Opfer und dem Antisemitismus in der österreichischen Nachkriegsgesellschaft verweist (Knight 1992: 221).

10 „Den Schritt aus der Anonymität ins Rampenlicht der Öffentlichkeit drückte die Israelitische Kultusgemeinde durch die gemeinsam mit der Stadt Wien und dem 'Jewish Welcome Service' organisierte Ausstellung 'Versunkene Welt' (1984), dem Besuch des New Yorker Bürgermeisters Edward Koch (1984), der Eröffnung des Chajes Gymnasiums (1984) oder mit der 1985 in Wien stattgefundenen Tagung des 'World Jewish Congress' aus." (Embacher 1995: 257) 
kann (vgl. Mitten 1992: 268), ${ }^{11}$ nicht auf Dauer erschüttern ließ (vgl. Embacher 1995: 260).

Ende der achtziger Jahre konnte die Israelitische Kultusgemeinde die bisher vergeblich beanspruchte Rolle einer moralischen Instanz gewinnen, was nicht nur auf den - sowohl auf jüdischer als auch auf nichtjüdischer Seite stattgefundenen-Generationswechsel, sondern hauptsächlich auf das in den achtziger Jahren angeschlagene Opferimage Österreichs zurückzuführen ist. (Ebd.: 261)

Auch Evelyn Adunka hebt die Veränderung innerhalb der jüdischen Gemeinde Wiens wie auch in ihren Beziehungen zum nicht-jüdischen Wien hervor, die Ende der 1980er Jahre vollzogen war. Sie berichtet über zahlreiche Veranstaltungen, die diese neue Selbstsicherheit ausdrückten.

1990 fand erstmals ein jüdisches Straßenfest mit Musik, Ausstellungen und Ständen aus Anlaß des Jom Jeruschalajim statt, das seither in der Umgebung des Stadttempels und später im Palais Lichtenstein alljährlich wiederholt wird. Eine selbstbewußte jüdische Gemeinde öffnete sich damit und zeigte sich ihrer Mitwelt in einer Form, wie es noch bis in die siebziger und achtziger Jahre völlig undenkbar gewesen wäre. (Adunka 2000: 527)

Dieses Selbstbewusstsein, das Auftreten in der Öffentlichkeit und der Zugewinn an öffentlicher Bedeutung, die wahrscheinlich sowohl auf den in Zweifel gezogenen Opfer-Status Österreichs als auch auf den Generationswechsel zurückzuführen sind, darf jedoch nicht über die Tatsache hinwegtäuschen, dass Antisemitismus nach wie vor weiter Existierte. Umfragen zufolge bejahten im Jahr 197321 Prozent die Frage, ob es besser sei, keine Juden im Land zu haben, im Jahr 1991 hingegen 15 Prozent der Befragten. Doch „1986 erklärten 35 Prozent der Befragten, sie stünden den Juden freundlich gegenüber, 1991 halbierte sich dieser Wert auf 18 Prozent, wohingegen sich die Ablehnung von sieben auf vierzehn Prozent verdoppelte." (Wassermann 2000: 312-313.)

Wenn die Uraufführung von Taboris Mein Kampf im Kontext dieses erstarkenden Selbstbewusstseins der jüdischen Gemeinde in Wien betrachtet wird, dann erscheint die Aufmerksamkeit, die ihr zuteilwurde, im neuen Licht. Die parodistische Darstellung Hitlers im ersten Teil des Stücks deutet auf eine selbstbewusste Position des Beobachters hin, der sich über den einst gefeierten und gefürchteten Diktator lustig machen kann. Es bedeutet nicht nur die

11 Ähnlich urteilt auch Matthias Konzett: „With Waldheim, finally, Austria’s silenced past became an open public theme and allowed writers to articulate their concerns with more national and international attention than ever before... Jewish themes and concerns now received more resonance in Austria." (Konzett 2000: 323) 
Entmythisierung Hitlers, sondern auch eine Aufwertung seiner Opfer. Noch wichtiger ist jedoch die Ablehnung der Festschreibung von Opferrollen, die Tabori bereits durch seine früheren Stücke veranschaulichte, die aber erst jetzt bei einem breiteren Publikum Gehör fand, das selbst die gesellschaftliche Last der Rollenzuschreibungen sowohl der Opfer als auch der Täter einsah und sich davon zu befreien suchte. Tabori leistete eine wichtige Vorarbeit mit seinen Stücken, die zum Teil bereits in den USA entstanden sind. Die geographische Distanz und die Erfahrung mit dem Rassismus und den Emanzipationsbewegungen in den USA verhalfen ihm schon viel früher zu den Einsichten, die in Wien erst Jahrzehnte später verstanden wurden. Doch obwohl der Augenblick, in dem die Uraufführung von Taboris nach langjährigem Schaffen ausgereiften Kunstwerk mit den gesellschaftlichen Prozessen in Mitteleuropa glücklich zusammenfiel, längst vergangen ist, so werden Rollen immer wieder von neuem zu- und -festgeschrieben, bestimmte Bevölkerungsgruppen kriminalisiert, andere viktimisiert. Zehn Jahre nach Taboris Tod und drei Jahrzehnte nach der Uraufführung von Mein Kampfließe sich dieses Werk wieder aktualisieren. Darüber hinaus sind seine Werke ein bleibender Beitrag zur literarischen Aufarbeitung von individuellen und kollektiven traumatischen Ereignissen.

\section{LITERATURVERZEICHNIS}

Adorno, Theodor W.: Ästhetische Theorie. Gesammelte Schriften, Bd. 7. Frankfurt am Main: Suhrkamp, 1970.

Adorno, Theodor W.: Kulturkritik und Gesellschaft. Gesammelte Schriften. B. 10. Frankfurt am Main: Suhrkamp, 1977.

Adorno, Theodor W.: Negative Dialektik. Gesammelte Schriften, Bd. 6. Frankfurt am Main: Suhrkamp, 1973.

Adunka, Evelyn: Die vierte Gemeinde. Die Geschichte der Wiener Juden von 1945 bis beute. Berlin, Wien: Philo, 2000.

Beller, Steven: Vienna and the Jews 1867 - 1938. A cultural history. Cambridge: Cambridge UP, 1989.

Berghahn, Klaus L.: „'Hitler und sein Jude': Anmerkungen zu George Taboris Mein Kampf". Modern Austrian Literature, Vol. 36, No. 1/2, 2003.

Dahlke, Karin: „Überrumpelte Katastrophen'. Taboris 'Witz' im Schatten der Shoah“. Bayerdörfer, Hans-Peter; Schönert, Jörg (Hg.). Theater gegen das Vergessen. Tübingen: Niemeyer, 1997. S. 123-154.

Embacher, Helga: Neubeginn ohne Illusionen. Juden in Österreich nach 1945. Wien: Picus, 1995.

Feinberg, Anat: George Tabori. München: DTV, 2003. 
Haas, Birgit: Das Theater des George Tabori: vom Verfremdungseffekt zur Postmoderne. Frankfurt am Main, Wien: Lang, 2000.

Höyng, Peter: „Immer spielt ihr und scherzt?“ Zur Dialektik des Lachens in George Taboris Mein Kampf. Farce.“ Peter Höyng (Hg.). Verkörperte Geschichtsentwürfe. Tübingen: Francke, 1998.

Huth, Alice: "In meiner Geisterstunde«. Intertextualität und Gedächtnis in Werken von George Tabori. Marburg: Tectum, 2008.

Klüger, Ruth: Weiter leben: eine Jugend. Göttingen: Wallstein, 1992.

Knight, Robert: “'Neutrality' Not Sympathy: Jews in Post-War Austria”. Robert S. Wistrich (Hg.). Austrians and Jews in the Twentieth Century. From Franz Joseph to Waldheim. New York: St. Martin's Press, 1992. S. 220-233.

Konzett, Matthias: "Transcultural Profiles in Contemporary Austrian Jewish Literature". Willy Riemer (Hg.): After Postmodernism. Austrian Literature and Film in Transition. Riverside, California: Ariadne Press, 2000. S. 315-339.

Kunne, Andrea: „Jüdische Identität in der österreichischen Nachkriegsliteratur. Peter Henisch, Robert Schinder, Robert Menasse und Doron Rabinovici.“ Klaus Hödl (Hg.): Jüdische Identitäten. Innsbruck, Wien, München: Studien V., 2000. S. 271-306.

Liessmann, Konrad Paul: „Die Tragödie als Farce: Anmerkungen zu George Taboris 'Mein Kampf'“. Text+Kritik, Heft 133, Januar 1997. S. 81-89.

Mitten, Richard: "New Faces of Anti-Semitic Prejudice in Austria: Reflections on the 'Waldheim Affair". Robert S. Wistrich (Hg.). Austrians and Jews in the Twentieth Century. From Franz Joseph to Waldheim. New York: St. Martin's Press, 1992. S. 252-273.

Oxaal, Ivar: "The Jews of Young Hitler's Vienna: Historical and Sociological Aspects". Ivar Oxaal, Michael Pollak, Gerhard Botz (Hg.): Jerws, Antisemitism and Culture in Vienna. London and New York: Routledge \& Kegan Paul, 1987. S. 11-38.

Pott, Sandra: „Ecce Schlomo’: Mein Kampf - Farce oder theologischer Schwank?"“. Bayerdörfer, Hans-Peter; Schönert, Jörg (Hg.). Theater gegen das Vergessen. Tübingen: Niemeyer, 1997. S. 248-269.

Roth, Markus: Theater nach Auschwitz: George Taboris Die Kannibalen im Kontext der Holocaust-Debatten. Frankfurt am Main: Lang, 2003.

Rozenblit, Marsha L.: "The Jews of Germany and Austria: A Comparative Perspective". Robert S. Wistrich (Hg.). Austrians and Jews in the Twentieth Century. From Franz Joseph to Waldheim. New York: St. Martin's Press, 1992. S. 1-18.

Rozenblit, Marsha L.: Die Juden Wiens 1867 - 1914. Assimilation und Identität. Wien, Köln, Graz: Böhlau, 1989.

Scholz, Stefan: Von der humanisierenden Kraft des Scheiterns. Stuttgart: Kohlhammer, 2002. 
Schubert, Kurt: Die Geschichte des österreichischen Judentums. Wien, Köln, Weimar: Böhlau, 2008.

Silverman, Lisa: Becoming Austrians. Jews and Culture between the World Wars. Oxford: Oxford UP, 2012.

Strümpel, Jan: Vorstellungen vom Holocaust: George Taboris Erinnerungs-Spiele. Göttingen: Wallstein, 2000.

Tabori, George: Theaterstücke II. München, Wien: Hanser, 1994.

Thunecke, Jörg: "Farce oder Volksstück? Eine Untersuchung der Theaterversion von George Taboris Mein Kampf”. Modern Austrian Literature, Vol. 26, No. 3/4, 1993. S. 247-272.

Wassermann, Heinz P.: „Eine Wohn-, aber keine Lebensgemeinschaft? Notizen zum Verhältnis zwischen nichtjüdischen und jüdischen Österreichern nach 1945“. Klaus Hödl (Hg.). Jüdische Identitäten. Innsbruck, Wien, München: Studien V., 2000. S. 307-334.

Wistrich, Robert S.: "The Kreisky Phenomenon: A Reassessment." Robert S. Wistrich (Hg.). Austrians and Jews in the Twentieth Century. From Franz Joseph to Waldheim. New York: St. Martin's Press, 1992. S. 234-251.

Zohn, Harry: „Wien erfüllt die Prophezeiung: Die Stadt ohne Juden“. Jüdisches Echo, 5. Jänner 1956.

Špela Virant

Universität Ljubljana spela.virant@guest.arnes.si

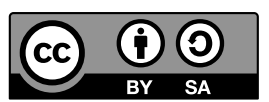

\section{Upodobljivost zla: literatura holokavsta Georgea Taborija}

Prispevek z medkulturnega vidika obravnava dramsko delo Georgea Taborija,

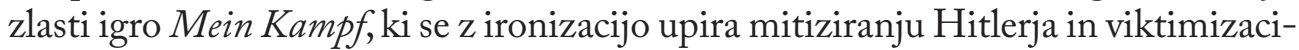
ji Judov, v kontekstu judovsko-nemških odnosov v Avstriji po drugi svetovni vojni. Igra je bila prvič uprizorjena leta 1987 na Dunaju, v času, ko je status Avstrije kot žrtve nacizma postajal vprašljiv, Judje na Dunaju pa so prvič po vojni spet začeli pridobivati samozavest.

Ključne besede: George Tabori, dramatika, holokavst, Judje na Dunaju, ironija 


\section{The Representability of Evil: George Tabori's Holocaust-Literature}

The article tries to read the plays by George Tabori from an intercultural point of view. The focus is on his play Mein Kampf, in which he uses irony to oppose the threatening mythologization of Hitler. It was first staged in 1987 in Vienna, when the status of Austria as a victim of Nazi-Germany became questionable and the Jews in Vienna regained their self-awareness for the first time after World War II.

Key words: George Tabori, Drama, Holocaust-Literature, Jews in Vienna, irony

Der Beitrag ist im Rahmen des Forschungsprogramms Interkulturelle literarurwisseschaftliche Studien (P6-0265) entstanden; die Autorin erkennt die finanzielle Unterstützung seitens der Slowenischen Forschungsagentur an. 